Research Article

\title{
Hyperstability of the $k$-Cubic Functional Equation in Non-Archimedean Banach Spaces
}

\author{
Youssef Aribou ${ }^{1}$ and Mohamed Rossafi (iD ${ }^{2}$ \\ ${ }^{1}$ Laayoune Higher School of Technology, Ibn Zohr University, BP 3007 Laayoune, Morocco \\ ${ }^{2}$ Department of Mathematics, University of Ibn Tofail, BP 133 Kenitra, Morocco \\ Correspondence should be addressed to Mohamed Rossafi; rossafimohamed@gmail.com
}

Received 28 August 2020; Revised 5 October 2020; Accepted 15 October 2020; Published 12 December 2020

Academic Editor: Ji Gao

Copyright (c) 2020 Youssef Aribou and Mohamed Rossafi. This is an open access article distributed under the Creative Commons Attribution License, which permits unrestricted use, distribution, and reproduction in any medium, provided the original work is properly cited.

Using the fixed point approach, we investigate a general hyperstability results for the following $k$-cubic functional equations $f(k x+y)+f(k x-y)=k f(x+y)+k f(x-y)+2 k\left(k^{2}-1\right) f(x)$, where $k$ is a fixed positive integer $\geq 2$, in ultrametric Banach spaces.

\section{Introduction}

The starting point of studying the stability of functional equations seems to be the famous talk of Ulam [1] in 1940, in which he discussed a number of important unsolved problems. Among those was the question concerning the stability of group homomorphisms.

Ulam's problem: let $G_{1}$ be a group and let $G_{2}$ be a metric group with a metric $d$. Given $\varepsilon>0$, there exists $\delta>0$ such that if a mapping $h: G_{1} \longrightarrow G_{2}$ satisfies the inequality

$$
d(h(x y), h(x) h(y))<\delta,
$$

for all $x, y \in G_{1}$, then there exists a homomorphism $H: G_{1} \longrightarrow G_{2}$ with

$$
d(h(x), H(x))<\varepsilon,
$$

for all $x \in G_{1}$.

The first partial answer, in the case of Cauchy equation in Banach spaces, to Ulam question was given by Hyers [2]. Later, the result of Hyers was first generalized by Aoki [3], and only much later by Rassias [4] and Găvruța [5]. Since then, the stability problems of several functional equations have been extensively investigated [6-10].

We say a functional equation is hyperstable if any function $f$ satisfying the equation approximately (in some sense) must be actually a solution to it. It seems that the first hyperstability result was published in [11] and concerned the ring homomorphisms. However, the term hyperstability has been used for the first time in [12]. Quite often, hyperstability is confused with superstability, which also admits bounded functions. Numerous papers on this subject have been published, and we refer, for example, to [3, 12-29].

Throughout this paper, $\mathbb{N}$ stands for the set of all positive integers and $N_{m_{0}}$, the set of integers greater than or equal $m_{0}$, $\mathbb{R}_{+}:=[0, \infty)$, and we use the notation $X_{0}$ for the set $X \backslash\{0\}$.

Let us recall (see, for instance, [30]) some basic definitions and facts concerning non-Archimedean normed spaces.

Definition 1. By a non-Archimedean field, we mean a field $\mathbb{K}$ equipped with a function (valuation) $|\cdot|: \mathbb{K} \longrightarrow[0, \infty)$ such that, for all $r, s \in \mathbb{K}$, the following conditions hold:

(1) $|r|=0$ if and only if $r=0$

(2) $|r s|=|r||s|$

(3) $|r+s| \leq \max \{|r|,|s|\}$

The pair $(\mathbb{K},||$.$) is called a valued field.$

In any non-Archimedean field, we have $|1|=|-1|=1$ and $|n| \leq 1$, for $n \in \mathbb{N}_{0}$. In any field $\mathbb{K}$, the function $|\cdot|: \mathbb{K} \longrightarrow \mathbb{R}_{+}$given by 


$$
|x|:= \begin{cases}0, & x=0, \\ 1, & x \neq 0,\end{cases}
$$

is a valuation which is called trivial, but the most important examples of non-Archimedean fields are $p$-adic numbers which have gained the interest of physicists for their research in some problems coming from quantum physics, $p$-adic strings, and superstrings.

Definition 2. Let $X$ be a vector space over a scalar field $\mathbb{K}$ with a non-Archimedean nontrivial valuation $|\cdot|$. A function $\|\cdot\|_{*}: X \longrightarrow \mathbb{R}$ is a non-Archimedean norm (valuation) if it satisfies the following conditions:

(1) $\|x\|_{*}=0$ if and only if $x=0$,

(2) $\|r x\|_{*}=|r|\|x\|_{*}(r \in \mathbb{K}, x \in X)$,

(3) The strong triangle inequality (ultrametric), namely,

$$
\|x+y\|_{*} \leq \max \left\{\|x\|_{*},\|y\|_{*}\right\}, \quad x, y \in X .
$$

Then, $\left(X,\|\cdot\|_{*}\right)$ is called a non-Archimedean normed space or an ultrametric normed space.

Definition 3. Let $\left\{x_{n}\right\}$ be a sequence in a non-Archimedean normed space $X$.

(1) A sequence $\left\{x_{n}\right\}_{n=1}^{\infty}$ in a non-Archimedean space is $a$ Cauchy sequence iff the sequence $\left\{x_{n+1}-x_{n}\right\}_{n=1}^{\infty}$ converges to zero.

(2) The sequence $\left\{x_{n}\right\}$ is said to be convergent if there exists $x \in X$ such that, for any $\varepsilon>0$, there is a positive integer $N$ such that $\left\|x_{n}-x\right\|_{*} \leq \varepsilon$, for all $n \geq N$. Then, the point $x \in X$ is called the limit of the sequence $\left\{x_{n}\right\}$, which is denoted by $\lim _{n \longrightarrow \infty} x_{n}=x$.

(3) If every Cauchy sequence in $X$ converges, then the non-Archimedean normed space $X$ is called a nonArchimedean Banach space or an ultrametric Banach space.

Let $X$ and $Y$ be normed spaces. A function $f: X \longrightarrow Y$ is called a $k$-cubic function provided it satisfies the functional equation:

$$
\begin{aligned}
f(k x+y)+f(k x-y)= & k f(x+y)+k f(x-y) \\
+ & 2 k\left(k^{2}-1\right) f(x), \\
& \text { for all } x, y \in X,
\end{aligned}
$$

and we can say that $f: X \longrightarrow Y$ is $k$-cubic on $X_{0}$ if it satisfies (5) for all $x, y \in X_{0}$.

In 2013, Bahyrycz et al. [31] used the fixed point theorem from Theorem 1 in [24] to prove the stability results for the generalization of $p$-Wright affine equation in ultrametric spaces. Recently, corresponding results for more general functional equations (in classical spaces) have been proved in [32-35].

In this paper, by using the fixed point method derived from $[20,21,36]$, we present some hyperstability results for equation (5) in ultrametric Banach spaces. Before proceeding to the main results, we state Theorem 1 which is useful for our purpose. To present it, we introduce the following three hypotheses:

(H1): $X$ is a nonempty set, $Y$ is an ultrametric Banach space over a non-Archimedean field, $f_{1}, \ldots, f_{k}$ : $X \longrightarrow X$, and $L_{1}, \ldots, L_{k}: X \longrightarrow \mathbb{R}_{+}$are given.

$(\mathrm{H} 2): \mathscr{T}: Y^{X} \longrightarrow Y^{X}$ is an operator satisfying the inequality

$$
\begin{aligned}
& \|\mathscr{T} \xi(x)-\mathscr{T} \mu(x)\|_{*} \\
& \leq \max _{1 \leq i \leq k}\left\{L_{i}(x)\left\|\xi\left(f_{i}(x)\right)-\mu\left(f_{i}(x)\right)\right\|_{*}\right\}, \\
& \quad \xi, \mu \in Y^{X}, x \in X .
\end{aligned}
$$

(H3): $\Lambda: \mathbb{R}_{+}^{X} \longrightarrow \mathbb{R}_{+}^{X}$ is a linear operator defined by

$$
\Lambda \delta(x):=\max _{1 \leq i \leq k}\left\{L_{i}(x) \delta\left(f_{i}(x)\right)\right\}, \quad \delta \in \mathbb{R}_{+}^{X}, x \in X .
$$

Thanks to a result due to Brzdçk and Ciepliński ([25], Remark 2), we state a slightly modified version of the fixed point theorem ([24], Theorem 1) in ultrametric spaces. We use it to assert the existence of a unique fixed point of operator $\mathscr{T}: Y^{X} \longrightarrow Y^{X}$.

Theorem 1. Let hypotheses (H1)-(H3) be valid, and functions $\varepsilon: X \longrightarrow \mathbb{R}_{+}$and $\varphi: X \longrightarrow Y$ fulfill the following two conditions:

$$
\begin{aligned}
\|\mathscr{T} \varphi(x)-\varphi(x)\|_{*} \leq \mathcal{E}(x), & x \in X, \\
\lim _{n \longrightarrow \infty} \Lambda^{n} \mathcal{\varepsilon}(x)=0, & x \in X .
\end{aligned}
$$

Then, there exists a unique fixed point $\psi \in Y^{X}$ of $\mathscr{T}$ with

$$
\|\varphi(x)-\psi(x)\|_{*} \leq \sup _{n \in \mathbb{N}_{0}} \Lambda^{n} \varepsilon(x), \quad x \in X .
$$

Moreover,

$$
\psi(x):=\lim _{n \longrightarrow \infty} \mathscr{T}^{n} \varphi(x), \quad x \in X .
$$

\section{Main Results}

In this section, we use Theorem 1 as a basic tool to prove the hyperstability results of the $k$-cubic functional equation (5) in ultrametric Banach spaces.

Theorem 2. Let $(X,\|\cdot\|)$ and $\left(Y,\|\cdot\|_{*}\right)$ be normed space and ultrametric Banach space, respectively, $c \geq 0, p, q \in \mathbb{R}$, and $p+q<0$, and let $f: X \longrightarrow Y$ satisfies

$$
\begin{aligned}
& \| f(k x+y)+f(k x-y)-k f(x+y)-k f(x-y) \\
& \quad-2\left(k^{3}-k\right) f(x)\left\|_{*} \leq c\right\| x\left\|^{p}\right\| y \|^{q},
\end{aligned}
$$

for all $x, y \in X_{0}$ such that $k x+y \neq 0, k x-y \neq 0, x+y \neq 0$, and $x-y \neq 0$. Then, $f$ is $k$-cubic on $X_{0}$. 
Proof. Take $m \in \mathbb{N}$ such that

$$
\alpha_{m}:=\left(\frac{m+1}{k}\right)^{p+q}<1, \quad m \geq k .
$$

Since $p+q<0$, one of $p, q$ must be negative. Assume that $q<0$ and replacing $y$ by $m x$ and $x$ by $((m+1) / k) x$ in (11), we obtain

$$
\begin{aligned}
& \left\|2\left(k^{3}-k\right) f\left(\frac{m+1}{k} x\right)+k f\left(\frac{k m+m+1}{k} x\right)+k f\left(\frac{m-k m+1}{k} x\right)-f((2 m+1) x)-f(x)\right\|_{*} \\
& \quad \leq c m^{q}\left(\frac{m+1}{k}\right)^{p}\|x\|^{p+q} .
\end{aligned}
$$

Define operators $\quad \mathscr{T}_{m}: Y^{X_{0}} \longrightarrow Y^{X_{0}} \quad$ and $\Lambda_{m}: \mathbb{R}_{+}^{X_{0}} \longrightarrow \mathbb{R}_{+}^{X_{0}}$ by

$$
\begin{aligned}
& \mathscr{T}_{m} \xi(x):=2\left(k^{3}-k\right) \xi\left(\frac{m+1}{k} x\right)+k \xi\left(\frac{k m+m+1}{k} x\right)+2 \xi\left(\frac{m-k m+1}{k} x\right)-\xi((2 m+1) x), \quad \xi \in Y^{X_{0}}, x \in X_{0}, \\
& \Lambda_{m} \delta(x):=\max \left\{\delta\left(\frac{m+1}{k} x\right), \delta\left(\frac{k m+m+1}{k} x\right), \delta\left(\left(\frac{m-k m+1}{k}\right) x\right), \delta(2 m+1)\right\}, \quad \delta \in \mathbb{R}_{+}^{X_{0}}, x \in X_{0},
\end{aligned}
$$

and write

$$
\varepsilon_{m}(x):=c m^{q}\left(\frac{m+1}{k}\right)^{p}\|x\|^{p+q}, \quad x \in X_{0} .
$$

It is easily seen that $\Lambda_{m}$ has the form described in (H3) with $k=4, \quad f_{1}(x)=((m+1) / k) x, \quad f_{2}(x)=((k m+$ $m+1) / k) x, \quad f_{3}(x)=((m-k m+1) / k) x, f_{4}(x)=(2 m+1) x$, and $L_{1}(x)=L_{2}(x)=L_{3}(x)=L_{4}(x)=1$. Furthermore, (11) can be written in the following way:

$$
\left\|\mathscr{T}_{m} f(x)-f(x)\right\|_{*} \leq \varepsilon_{m}(x), \quad x \in X_{0} .
$$

Moreover, for every $\xi, \mu \in Y^{X_{0}}, x \in X_{0}$,

$$
\begin{aligned}
& \left\|\mathscr{T}_{m} \xi(x)-\mathscr{T}_{m} \mu(x)\right\|_{*}=\| 2\left(k^{3}-k\right) \xi\left(\left(\frac{m+1}{k}\right) x\right)+k \xi\left(\left(\frac{k m+m+1}{k}\right) x\right)+k \xi\left(\left(\frac{m-k m+1}{k}\right) x\right)- \\
& \xi((2 m+1) x)-2\left(k^{3}-k\right) \mu\left(\left(\frac{m+1}{k}\right) x\right)-k \mu\left(\left(\frac{k m+m+1}{k}\right) x\right)-k \mu\left(\left(\frac{m-k m+1}{k}\right) x\right)+\xi((2 m+1) x) \|_{*} \\
& \quad \leq \max \left\{\left\|\xi\left(\left(\frac{m+1}{k}\right) x\right)-\mu\left(\left(\frac{m+1}{k}\right) x\right)\right\|_{*},\left\|\xi\left(\frac{k m+m+1}{k} x\right)-\mu\left(\frac{k m+m+1}{k} x\right)\right\|_{*}\right. \\
& \left.\left.\left\|\xi\left(\frac{m-k m+1}{K} x\right)-\mu\left(\frac{m-k m+1}{k} x\right)\right\|_{*}, \| \xi(2 m+1) x\right)-\mu((2 m+1) x) \|_{*}\right\} .
\end{aligned}
$$

So, (H2) is valid.

By using mathematical induction, we will show that, for each $x \in X_{0}$, we have

$$
\Lambda_{m}^{n} \varepsilon_{m}(x)=c m^{q}\left(\frac{m+1}{k}\right)^{p}\|x\|^{p+q} \alpha_{m}^{n},
$$

where $\alpha_{m}=((m-1) / k)^{p+q}$. We obtain that (18) holds for $n=0$. Next, we will assume that (18) holds for $n=r$, where $r \in \mathbb{N}$. Then, we have 


$$
\begin{aligned}
\Lambda_{m}^{r+1} \varepsilon_{m}(x)= & \Lambda_{m}\left(\Lambda_{m}^{r} \varepsilon_{m}(x)\right) \\
= & \max \left\{\Lambda_{m}^{r} \varepsilon_{m}\left(\frac{m+1}{k} x\right), \Lambda_{m}^{r} \varepsilon_{m}\left(\frac{k m+m+1}{k} x\right), \Lambda_{m}^{r} \varepsilon_{m}\left(\frac{k m-m-1}{k} x\right), \Lambda_{m}^{r} \varepsilon_{m}((2 m+1) x)\right\} \\
= & \max \left\{c m^{q}\left(\frac{m+1}{k}\right)^{p}\|x\|^{p+q} \alpha_{m}^{r}\left(\frac{m+1}{k}\right)^{p+q}, c m^{q}\left(\frac{m+1}{k}\right)^{p}\|x\|^{p+q} \alpha_{m}^{r}\left(\frac{k m+m+1}{k}\right)^{p+q},\right. \\
& \left.c m^{q}\left(\frac{m+1}{K}\right)^{p}\|x\|^{p+q} \alpha_{m}^{r}\left(\frac{k m-m-1}{k}\right)^{p+q}, c m^{q}\|x\|^{p+q} \alpha_{m}^{k}(2 m+1)^{p+q}\right\} \\
= & c m^{q}\|x\|^{p+q} \alpha_{m}^{r} \max \left\{\left(\frac{m+1}{k}\right)^{p+q},\left(\frac{k m+m+1}{k}\right)^{p+q},\left(\frac{k m-m-1}{k}\right)^{p+q},(2 m+1)^{p+q}\right\} \\
= & c m^{q}\left(\frac{m+1}{k}\right)^{p}\|x\|^{p+q} \alpha_{m}^{r+1}, x \in X_{0} .
\end{aligned}
$$

This shows that (18) holds for $n=r+1$. Now, we can conclude that inequality (18) holds for all $n \in \mathbb{N}_{0}$. From (18), we obtain

$$
\lim _{n \rightarrow \infty} \Lambda^{n} \varepsilon_{m}(x)=0
$$

for all $x \in X_{0}$.

Hence, according to Theorem 1, there exists a unique solution $C_{m}: X_{0} \longrightarrow Y$ of the equation:

$$
C_{m}(x)=2\left(k^{3}-k\right) C_{m}\left(\frac{m+1}{k} x\right)+k C_{m}\left(\frac{k m+m+1}{k} x\right)+k C_{m}\left(\frac{1-k m+m}{k} x\right)-C_{m}((2 m+1) x), \quad x \in X_{0}
$$

such that

Moreover,

$$
\left\|f(x)-C_{m}(x)\right\|_{*} \leq \sup _{n \in \mathbb{N}_{0}}\left\{c m^{q}\left(\frac{m+1}{k}\right)^{p}\|x\|^{p+q} \alpha_{m}^{n}\right\}, \quad x \in X_{0} .
$$

$$
C_{m}(x):=\lim _{n \longrightarrow \infty} \mathscr{T}_{m}^{n} f(x)
$$

for all $x \in X_{0}$.

Now, we show that

$$
\begin{aligned}
& \left\|\mathscr{T}_{m}^{n} f(k x+y)+\mathscr{T}_{m}^{n} f(k x-y)-k \mathscr{T}_{m}^{n} f(x+y)-k \mathscr{T}_{m}^{n} f(x-y)-2\left(k^{3}-k\right) \mathscr{T}_{m}^{n} f(x)\right\|_{*} \\
& \quad \leq c \alpha_{m}^{n}\|x\|^{p}\|y\|^{q},
\end{aligned}
$$

for every $x, y \in X_{0}$ such that $x+y \neq 0, x-y \neq 0$. Since the case $n=0$ is just (11), take $r \in \mathbb{N}$, and suppose the last inequality holds for $n=r$ and every $x, y \in X_{0}$ such that $x+y \neq 0, x-y \neq 0$. Then, 


$$
\begin{aligned}
& \left\|\mathscr{T}_{m}^{r+1} f(k x+y)+\mathscr{T}_{m}^{r+1} f(k x-y)-k \mathscr{T}_{m}^{r+1} f(x+y)-k \mathscr{T}_{m}^{r+1} f(x-y)-2\left(k^{3}-k\right) \mathscr{T}_{m}^{r+1} f(x)\right\|_{*} \\
& =\| 2\left(k^{3}-k\right) \mathscr{T}_{m}^{k} f\left(\frac{m+1}{k}(k x+y)\right)+k \mathscr{T}_{m}^{r} f\left(\frac{k m+m+1}{k}(k x+y)\right)+k \mathscr{T}_{m}^{r} f\left(\frac{1+m-k m}{k}(k x+y)\right) \\
& \left.-\mathscr{T}_{m}^{r} f((2 m+1)(k x+y))+2\left(k^{3}-k\right]\right) \mathscr{T}_{m}^{r} f\left(\frac{m+1}{k}(k x-y)\right)+k \mathscr{T}_{m}^{r} f\left(\frac{k m+m+1}{k}(k x-y)\right) \\
& +k \mathscr{T}_{m}^{r} f\left(\frac{1+m-k m}{k}(k x-y)\right)-\mathscr{T}_{m}^{r} f((2 m+1)(k x-y))-2 k\left(k^{3}-k\right) \mathscr{T}_{m}^{r} f\left(\frac{m+1}{k}(x+y)\right) \\
& -k^{2} \mathscr{T}_{m}^{r} f\left(\frac{k m+m+1}{k}(x+y)\right)-k^{2} \mathscr{T}_{m}^{r} f\left(\frac{1+m-k m}{k}(x+y)\right)+k \mathscr{T}_{m}^{r} f((2 m+1)(x+y)) \\
& -2 k\left(k^{3}-k\right) \mathscr{T}_{m}^{r} f\left(\frac{m+1}{k}(x-y)\right)-k^{2} \mathscr{T}_{m}^{r} f\left(\frac{k m+m+1}{k}(x-y)\right)-k^{2} \mathscr{T}_{m}^{r} f\left(\frac{1+m-k m}{k}(x-y)\right) \\
& +\mathscr{T}_{m}^{r} f((2 m+1)(x-y))-\left(2\left(k^{3}-k\right)\right)^{2} \mathscr{T}_{m}^{r} f\left(\left(\frac{m+1}{k}\right)(x)\right)-2 k\left(k^{3}-k\right) \mathscr{T}_{m}^{r} f\left(\left(\frac{k m+m+1}{k}\right)(x)\right) \\
& -2 k\left(k^{3}-k\right) \mathscr{T}_{m}^{r} f\left(\left(\frac{1+m-k m}{k}\right)(x)\right)+2\left(k^{3}-k\right) \mathscr{T}_{m}^{k} f\left(\left.(2 m+1)(x)\right|_{*}\right. \\
& \leq \max \left\{\| \mathscr{T}_{m}^{n} f\left(\frac{m+1}{k}(k x+y)\right)+\mathscr{T}_{m}^{n} f\left(\frac{m+1}{k}(k x-y)\right)-k \mathscr{T}_{m}^{n} f\left(\frac{m+1}{k}(x+y)\right)\right. \\
& -k \mathscr{T}_{m}^{n} f\left(\frac{m+1}{k}(x-y)\right)-2\left(k^{3}-k\right) \mathscr{T}_{m}^{n} f\left(\frac{m+1}{k} x\right) \|_{*}, \\
& \| \mathscr{T}_{m}^{n} f\left(\frac{k m+m+1}{k}(k x+y)\right)+\mathscr{T}_{m}^{n} f\left(\frac{k m+m+1}{k}(k x-y)\right)-k \mathscr{T}_{m}^{n} f\left(\frac{k m+m+1}{k}(x+y)\right) \\
& -k \mathscr{T}_{m}^{n} f\left(\frac{k m+m+1}{k}(x-y)\right)-2\left(k^{3}-k\right) \mathscr{T}_{m}^{n} f\left(\frac{k m+m+1}{k} x\right) \|_{*}, \\
& \| \mathscr{T}_{m}^{n} f\left(\frac{1+m-k m}{k}(k x+y)\right)+\mathscr{T}_{m}^{n} f\left(\frac{1+m-k m}{k}(k x-y)\right)-k \mathscr{T}_{m}^{n} f\left(\frac{1+m-k m}{k}(x+y)\right) \\
& -k \mathscr{T}_{m}^{n} f\left(\frac{1+m-k m}{k}(x-y)\right)-2\left(k^{3}-k\right) \mathscr{T}_{m}^{n} f\left(\frac{1+m-k m}{k} x\right) \|_{*}, \\
& \| \mathscr{T}_{m}^{n} f((2 m+1)(k x+y))+\mathscr{T}_{m}^{n} f((2 m+1)(k x-y))-k \mathscr{T}_{m}^{n} f((2 m+1)(x+y)) \\
& \left.-\mathscr{T}_{m}^{n} f((2 m+1)(x-y))-2\left(k^{3}-k\right) \mathscr{T}_{m}^{n} f((2 m+1) x) \|_{*}\right\} \\
& \leq \max \left\{c \alpha_{m}^{k}\|x\|^{p}\|y\|^{q}\left(\frac{m+1}{k}\right)^{p+q}, c \alpha_{m}^{k}\|x\|^{p}\|y\|^{q}\left(\frac{k m+m+1}{k}\right)^{p+q},\right. \\
& \left.c \alpha_{m}^{k}\|x\|^{p}\|y\|^{q}\left(\frac{1+m-k m}{k}\right)^{p+q}, c \alpha_{m}^{k}\|x\|^{p}\|y\|^{q}(2 m+1)^{p+q}\right\} \\
& =c \alpha_{m}^{k}\|x\|^{p}\|y\|^{q} \max \left\{\left(\frac{m+1}{k}\right)^{p+q},\left(\frac{k m+m+1}{k}\right)^{p+q},\left(\frac{k m-m-1}{k}\right)^{p+q},(k m+m+1)^{p+q}\right\} \\
& \leq c \alpha_{m}^{k+1}\|x\|^{p}\|y\|^{q},
\end{aligned}
$$


for all $x, y \in X_{0}$ such that $x+y \neq 0$ and $x-y \neq 0$. Thus, by induction, we have shown that suppose the last inequality holds for every $n \in \mathbb{N}_{0}$. Letting $n \longrightarrow \infty$, we obtain that

$$
\begin{aligned}
C_{m}(k x+y)+C_{m}(k x-y)= & k C_{m}(x+y)+k C_{m}(x-y) \\
& +2\left(k^{3}-k\right) C_{m}(x),
\end{aligned}
$$

for all $x, y \in X_{0}$ such that $x+y \neq 0, x-y \neq 0$. In this way, we obtain a sequence $\left\{C_{m}\right\}_{m \geq m_{0}}$ of $k$-cubic functions on $X_{0}$ such that

$$
\begin{array}{r}
\left\|f(x)-C_{m}(x)\right\|_{*} \leq \sup _{n \in \mathbb{N}_{0}}\left\{c m^{q}\left(\frac{m+1}{k}\right)^{p}\|x\|^{p+q} \alpha_{m}^{n}\right\}, \\
x \in X_{0} .
\end{array}
$$

This implies that

$$
\left\|f(x)-C_{m}(x)\right\|_{*} \leq c m^{q}\left(\frac{m+1}{k}\right)^{p}\|x\|^{p+q}, \quad x \in X_{0} .
$$

It follows, with $m \longrightarrow \infty$, that $f$ is $k$-cubic on $X_{0}$. In a similar way, we can prove the following theorem.

Theorem 3. Let $(X,\|\cdot\|)$ and $\left(Y,\|\cdot\|_{*}\right)$ be normed space and ultrametric Banach space, respectively, $c \geq 0, p, q \in \mathbb{R}$, and $p+q>0$, and let $f: X \longrightarrow Y$ satisfies

$$
\left\|f(k x+y)+f(k x-y)-k f(x+y)-k f(x-y)-2\left(k^{3}-k\right) f(x)\right\|_{*} \leq c\|x\|^{p}\|y\|^{q},
$$

for all $x, y \in X_{0}$ such that $x+y \neq 0$ and $x-y \neq 0$. Then, $f$ is $k$-cubic on $X_{0}$.

Proof. Take $m \in \mathbb{N}$ such that

$$
\alpha_{m}=\left(\frac{m-4}{m}\right)^{p+q}<1
$$

$$
\begin{aligned}
& \left\|2\left(k^{3}-k\right) f\left(\frac{m-2}{k m} y\right)+k f\left(\frac{m+2}{k m} y\right)+k f\left(\frac{m-6}{k m} y\right)-f\left(\frac{m-4}{m} y\right)-f(y)\right\|_{*} \\
& \quad \leq c\left(\frac{2}{m}\right)^{q}\left(\frac{m-2}{k m}\right)^{p}\|y\|^{p+q}, \quad x \in X_{0} .
\end{aligned}
$$

Writing

$$
\begin{aligned}
\mathscr{T}_{m} \xi(x) & :=2\left(k^{3}-k\right) \xi\left(\frac{m-2}{k m} x\right)+k \xi\left(\frac{m+2}{k m} x\right)+k \xi\left(\frac{m-6}{k m} x\right)-\xi\left(\frac{m-4}{m} x\right), \quad \xi \in Y^{X_{0}}, x \in X_{0}, \\
\varepsilon_{m}(x) & :=c\left(\frac{2}{m}\right)^{q}\left(\frac{m-1}{k m}\right)^{p}\|x\|^{p+q}, \quad x \in X_{0},
\end{aligned}
$$

(31) takes form

$$
\left\|\mathscr{T}_{m} f(x)-f(x)\right\|_{*} \leq \varepsilon_{m}(x), \quad x \in X_{0} .
$$

The rest of the proof is similar to the proof of the last theorem. It easy to show the hyperstability of cubic equation on the set containing 0 . The above theorems imply, in particular, the following corollary, which shows their simple application.
Corollary 1. Let $(X,\|\cdot\|)$ and $\left(Y,\|\cdot\|_{*}\right)$ be normed space and ultrametric Banach space, respectively, $G: X^{2} \longrightarrow Y$ and $G\left(x_{0}, y_{0}\right) \neq 0$ for some $x_{0}, y_{0} \in X$, and

$$
\|G(x, y)\|_{*} \leq c\|x\|^{p}\|y\|^{q}, \quad x, y \in X,
$$

where $c \geq 0, p, q \in \mathbb{R}$. Assume that the numbers $p$ and $q$ satisfy one of the following conditions:

(1) $p+q<0$, and (11) holds for all $x, y \in X_{0}$ 
(2) $p+q>0$, and (36) holds for all $x, y \in X_{0}$

Then, the functional equation

$$
\begin{aligned}
& f(k x+y)+f(k x+y) \\
& \quad=k f(x+y)+k f(x-y) \\
& \quad+2\left(k^{3}-k\right) f(x)+G(x, y), \quad x, y \in X,
\end{aligned}
$$

has no solution in the class of functions $g: X \longrightarrow Y$.

In the following theorem, we present a general hyperstability for the $k$-cubic equation where the control function is $\varphi(x)+\varphi(y)$, which corresponds to the approach introduced in [36].
Theorem 4. Let $(X,\|\cdot\|)$ be a normed space, $\left(Y,\|\cdot\|_{*}\right)$ be an ultrametric Banach space over a field $\mathbb{K}$, and $\varphi: X \longrightarrow \mathbb{R}_{+}$be a function such that

$$
\begin{aligned}
U:= & \left\{n \in \mathbb{N}: \alpha_{n}:=\max \{\lambda(n), \lambda(k n+n-1),\right. \\
& \lambda(-k n+n+1), \lambda(2 k n-1)\}<1\},
\end{aligned}
$$

is a nonempty set, where $\lambda(a):=\inf \left\{t \in \mathbb{R}_{+}\right.$: $\varphi(a x) \leq t \varphi(x)$ for $x \in X\}$ for all $a \in \mathbb{K}_{0}$. Suppose that

$$
\begin{aligned}
& \lim _{a \longrightarrow \infty} \lambda(a)=0, \\
& \lim _{a \longrightarrow \infty} \lambda(-a)=0,
\end{aligned}
$$

and $f: X \longrightarrow Y$ satisfies the inequality

$$
\left\|f(k x+y)+f(k x+y)-k f(x+y)-k f(x-y)-2\left(k^{3}-k\right) f(x)\right\|_{*} \leq \varphi(x)+\varphi(y),
$$

for all $x, y \in X_{0}$, such that $x+y \neq 0$ and $x-y \neq 0$. Then, $f$ is cubic on $X_{0}$.
Proof. Replacing $x$ by $m x$ and $y$ by $(-k m+1) x$ for $m \in \mathbb{N}$ in (39), we get

$$
\begin{aligned}
& \left\|f(x)+f((2 k m-1) x)-k f((-k m+m+1) x)-k f((k m+m-1) x)-2\left(k^{3}-k\right) f(m x)\right\|_{*} \\
& \quad \leq \varphi((-k m+1) x)+\varphi(m x),
\end{aligned}
$$

for all $x \in X_{0}$. For each $m \in U$, we define the operator $\mathscr{T}_{m}: Y^{X_{0}} \longrightarrow Y^{X_{0}}$ by

$$
\begin{array}{r}
\mathscr{T}_{m} \xi(x):=2\left(k^{3}-k\right) \xi(m x)+k \xi((k m+m-1) x) \\
+k \xi((-k m+m+1) x)-\xi((2 k m-1) x), \\
\xi \in Y^{X_{0}}, x \in X_{0} .
\end{array}
$$

Furthermore, we put

$$
\begin{aligned}
\varepsilon_{m}(x):= & \varphi((-k m+1) x)+\varphi(m x) \leq(\lambda(-k m+1) \\
& +\lambda(m x)) \varphi(x), \quad x \in X_{0} .
\end{aligned}
$$

Then, inequality (40) takes the form

$$
\left\|\mathscr{T}_{m} f(x)-f(x)\right\|_{*} \leq \varepsilon_{m}(x), \quad x \in X_{0} .
$$

For each $m \in U$, the operator $\Lambda_{m}: \mathbb{R}_{+}^{X_{0}} \longrightarrow \mathbb{R}_{+}^{X_{0}}$ which is defined by $\Lambda_{m} \delta(x):=\max \{\delta(m x), \delta((k m+m-1) x)$, $\delta((-k m+m+1) x), \delta((2 k m-1) x)\}, \delta \in \mathbb{R}_{+}^{X_{0}}, x \in X_{0}$, has the form described in (H3) with $k=4$ and

$$
\begin{aligned}
& f_{1}(x)=m x, \\
& f_{2}(x)=(k m+m-1) x, \\
& f_{3}(x)=(-k m+m+1) x, \\
& f_{4}(x)=(2 k m-1) x, \\
& L_{1}(x)=L_{2}(x)=L_{2}(x)=L_{4}(x)=1,
\end{aligned}
$$

for all $x \in X_{0}$. Moreover, for every $\xi, \mu \in Y^{X_{0}}, x \in X_{0}$,

$$
\begin{aligned}
\left\|\mathscr{T}_{m} \xi(x)-\mathscr{T}_{m} \mu(x)\right\|_{*}= & \| 2\left(k^{3}-k\right) \xi((m) x)+k \xi((k m+m-1) x)+k \xi((-k m+m+1) x) \\
& -\xi((2 k m-1) x)-2\left(k^{3}-k\right) \mu((m) x)-k \mu((k m+m-1) x)-k \mu((-k m+m+1) x) \\
& +\xi((2 k m-1) x) \|_{*} \\
\leq & \max \left\{\|\xi((m) x)-\mu((m) x)\|_{*},\|\xi((k m+m-1) x)-\mu((k m+m-1) x)\|_{*},\right. \\
& \left.\|\xi((-k m+m+1) x)-\mu((-k m+m+1) x)\|_{*},\|\xi((2 k m-1) x)-\mu((2 k m-1) x)\|_{*}\right\} .
\end{aligned}
$$


So, (H2) is valid. By using mathematical induction, we will show that, for each $x \in X_{0}$, we have

$$
\Lambda_{m}^{n} \varepsilon_{m}(x) \leq(\lambda(-k m+1)+\lambda(m)) \alpha_{m}^{n} \varphi(x) .
$$

From (42), we obtain that inequality (46) holds for $n=0$. Next, we will assume that (46) holds for $n=r$, where $r \in \mathbb{N}$. Then, we have

$$
\begin{aligned}
\Lambda_{m}^{r+1} \varepsilon_{m}(x)= & \Lambda_{m}\left(\Lambda_{m}^{r} \varepsilon_{m}(x)\right)=\max \left\{\Lambda_{m}^{r} \varepsilon_{m}(m x), \Lambda_{m}^{r} \varepsilon_{m}((k m+m-1) x), \Lambda_{m}^{r} \varepsilon_{m}((-k m+m+1) x), \Lambda_{m}^{r} \varepsilon_{m}((2 k m-1) x)\right\} \\
& \leq(\lambda(m)+\lambda(-k m+1)) \alpha_{m}^{r} \max \{\varphi(m x), \varphi((k m+m-1) x), \varphi((-k m+m+1) x), \varphi((2 k m-1) x)\} \\
& \leq(\lambda(m)+\lambda(-k m+1)) \alpha_{m}^{r+1} \varphi(x) \\
& x \in X_{0} .
\end{aligned}
$$

This shows that (46) holds for $n=r+1$. Now, we can conclude that inequality (46) holds for all $n \in \mathbb{N}$. From (46), we obtain $\lim _{n \longrightarrow \infty} \Lambda^{n} \varepsilon_{m}(x)=0$, for all $x \in X_{0}$ and all $m \in U$. Hence, according to Theorem 1 , there exists, for each $m \in U$, a unique solution $C_{m}: X_{0} \longrightarrow Y$ of the equation:

$$
\begin{aligned}
C_{m}(x)= & 2\left(k^{3}-k\right) C_{m}(m x)+k C_{m}((k m+m-1) x) \\
& +k C_{m}((-k m+m+1) x)-C_{m}((2 k m-1) x),
\end{aligned}
$$

$x \in X_{0}$, such that

$$
\begin{array}{r}
\left\|f(x)-C_{m}(x)\right\|_{*} \leq \sup _{n \in \mathbb{N}_{0}}\left\{(\lambda(m)+\lambda(-k m+1)) \alpha_{m}^{n} \varphi(x)\right\}, \\
x \in X_{0} .
\end{array}
$$

Moreover, $C_{m}(x):=\lim _{n \longrightarrow \infty}\left(\mathscr{T}_{m}^{n} f\right)(x)$, for all $x \in X_{0}$. Now, we show that

$$
\begin{aligned}
& \left\|\mathscr{T}_{m}^{n} f(k x+y)+\mathscr{T}_{m}^{n} f(k x-y)-k \mathscr{T}_{m}^{n} f(x+y)-k \mathscr{T}_{m}^{n} f(x+y)-2\left(k^{3}-k\right) \mathscr{T}_{m}^{n} f(x)\right\|_{*} \\
& \quad \leq \alpha_{m}^{n}(\varphi(x)+\varphi(y)),
\end{aligned}
$$

for every $x, y \in X_{0}$ such that $x+y \neq 0, x-y \neq 0$, and $n \in \mathbb{N}$. Since the case $n=0$ is just (39), take $k \in \mathbb{N}$ and assume that
(50) holds for $n=r$, where $r \in \mathbb{N}$ and every $x, y \in X_{0}$ such that $x+y \neq 0$. Then,

$$
\begin{array}{rl}
\| \mathscr{T}_{m}^{r+1} & f(k x+y)+\mathscr{T}_{m}^{k+1} f(k x-y)-k \mathscr{T}_{m}^{r+1} f(x+y)-k \mathscr{T}_{m}^{r+1} f(x-y)-2\left(k^{3}-k\right) \mathscr{T}_{m}^{r+1} f(x) \|_{*} \\
= & \| 2\left(k^{3}-k\right) \mathscr{T}_{m}^{r} f(m(k x+y))+k \mathscr{T}_{m}^{r} f((k m+m-1)(k x+y)) \\
& +k \mathscr{T}_{m}^{r} f((-k m+m+1)(k x+y))-\mathscr{T}_{m}^{r} f((2 k m-1)(k x+y))+2\left(k^{3}-k\right) \mathscr{T}_{m}^{r} f((m)(k x-y)) \\
& +k \mathscr{T}_{m}^{r} f((k m+m-1)(k x-y))+k \mathscr{T}_{m}^{r} f((-k m+m+1)(k x-y))-\mathscr{T}_{m}^{r} f((k k m-1)(k x-y)) \\
& -k\left(k^{3}-k\right) \mathscr{T}_{m}^{r} f((m)(x+y))-k^{2} \mathscr{T}_{m}^{r} f((k m+m-1)(x+y))-k^{2} \mathscr{T}_{m}^{r} f((-k m+m+1)(x+y)) \\
& +k \mathscr{T}_{m}^{r} f((2 k m-1)(x+y))-k\left(k^{3}-k\right) \mathscr{T}_{m}^{r} f((m)(x-y))-k^{2} \mathscr{T}_{m}^{r} f((k m+m-1)(x-y)) \\
& -k^{2} \mathscr{T}_{m}^{r} f((-k m+m+1)(x-y)) k \mathscr{T}_{m}^{r} f((2 k m-1)(x-y))-\left(2\left(k^{3}-k\right)\right)^{2} \mathscr{T}_{m}^{r} f((m)(x)) \\
& -2 k\left(k^{3}-k\right) \mathscr{T}_{m}^{r} f((k m+m-1)(x))-2 k\left(k^{3}-k\right) \mathscr{T}_{m}^{r} f((-k m+m+1)(x)) \\
& +\left.2\left(k^{3}-k\right) \mathscr{T}_{m}^{r} f((2 k m-1)(x))\right|_{*} \\
\leq & \max \left\{\| \mathscr{T}_{m}^{n} f(m(k x+y))+\mathscr{T}_{m}^{n} f(m(k x-y))-k \mathscr{T}_{m}^{n} f(m(x+y))-k \mathscr{T}_{m}^{n} f(m(x-y))\right. \\
& -2\left(k^{3}-k\right) \mathscr{T}_{m}^{n} f(m x) \|_{*},
\end{array}
$$




$$
\begin{aligned}
& \| \mathscr{T}_{m}^{n} f((k m+m-1)(k x+y))+\mathscr{T}_{m}^{n} f((k m+m-1)(k x-y))-k \mathscr{T}_{m}^{n} f((k m+m-1)(x+y)) \\
& \quad-\mathscr{T}_{m}^{n} f((k m+m-1)(x-y))-2\left(k^{3}-k\right) \mathscr{T}_{m}^{n} f((k m+m-1) x) \|_{*}, \\
& \| \mathscr{T}_{m}^{n} f((m-k m+1)(k x+y))+\mathscr{T}_{m}^{n} f((m-k m+1)(k x-y))-k \mathscr{T}_{m}^{n} f((m-k m+1)(x+y)) \\
& \quad-\mathscr{T}_{m}^{n} f((m-k m+1)(x-y))-2\left(k^{3}-k\right) \mathscr{T}_{m}^{n} f((m-k m+1) x) \|_{*}, \\
& \| \mathscr{T}_{m}^{n} f((2 k m-1)(k x+y))+\mathscr{T}_{m}^{n} f((2 k m-1)(k x-y))-k \mathscr{T}_{m}^{n} f((2 k m-1)(x+y))- \\
& \quad \mathscr{T}_{m}^{n} f\left(((2 k m-1)(x-y))-2\left(k^{3}-k\right) \mathscr{T}_{m}^{n} f((2 k m-1) x) \|_{*}\right\}, \\
& \leq \max _{m}\left\{\alpha_{m}^{k}\left(\varphi\left((m x)+\varphi((m y)), \alpha_{m}^{k}(\varphi(k m+m-1) x)+\varphi((K m+m-1) y)\right)\right)\right. \\
& \left.\alpha_{m}^{k}((\varphi(-k m+m+1) x)+\varphi((-k m+m+1) y)), \alpha_{m}^{k}((\varphi(2 k m-1) x)+\varphi((2 k m-1) y))\right\} \\
& \quad \leq \alpha_{m}^{k} \max \{\lambda(m), \lambda(k m+m-1), \lambda(-k m+m+1), \lambda(2 k m-1)\}(\varphi(x)+\varphi(y)) \\
& \quad=\alpha_{m}^{k+1}(\varphi(x)+\varphi(y)) .
\end{aligned}
$$

Thus, by induction, we have shown that (50) holds for every $n \in \mathbb{N}$. Letting $n \longrightarrow \infty$ in (50), we obtain that

$$
\begin{aligned}
& C_{m}(k x+y)+C_{m}(k x-y) \\
& \quad=k C_{m}(x+y)+k C_{m}(x-y)+2\left(k^{3}-k\right) C_{m}(x),
\end{aligned}
$$

for all $x, y \in X_{0}$ such that $x+y \neq 0$ and $x-y \neq 0$. In this way, we obtain a sequence $\left\{C_{m}\right\}_{m \in U}$ of $k$-cubic functions on $X_{0}$ such that

$$
\begin{array}{r}
\left\|f(x)-C_{m}(x)\right\|_{*} \leq \sup _{n \in \mathbb{N}_{0}}\left\{(\lambda(m)+\lambda(-k m+1)) \alpha_{m}^{n} \varphi(x)\right\}, \\
x \in X_{0} .
\end{array}
$$

This implies that

$$
\left\|f(x)-C_{m}(x)\right\|_{*} \leq(\lambda(m)+\lambda(-k m+1)) \varphi(x), \quad x \in X_{0},
$$

because the precedent inequality holds for over $n=0$ and $\alpha_{m}<1$.

It follows, with $m \longrightarrow \infty$, that $f$ is cubic on $X_{0}$. The following corollary is a particular case of Theorem 4 where $\varphi(x):=c\|x\|^{p}$ with $c \geq 0$ and $p<0$.

Corollary 2. Let $(X,\|\cdot\|)$ and $\left(Y,\|\cdot\|_{*}\right)$ be normed space and ultrametric Banach space, respectively, $c \geq 0$, and $p<0$, and let $f: X \longrightarrow Y$ satisfies

$$
\left\|f(k x+y)+f(k x+y)-k f(x+y)-k f(x-y)-2\left(k^{3}-k\right) f(x)\right\|_{*} \leq c\left(\|x\|^{p}+\|y\|^{p}\right),
$$

for all $x, y \in X_{0}$. Then, $f$ is $k$-cubic on $X_{0}$.

\section{Data Availability}

No data were used to support this study.

\section{Conflicts of Interest}

The authors declare that they have no conflicts of interest.

\section{References}

[1] S. M. Ulam, Problems in Modern Mathematics, Science Editions, Wiley, New York, NY, USA, 1964.

[2] D. H. Hyers, "On the stability of the linear functional equation," Proceedings of the National Academy of Sciences, vol. 27, no. 4, pp. 222-224, 1941.
[3] T. Aoki, "On the stability of the linear transformation in Banach spaces," Journal of the Mathematical Society of Japan, vol. 2, no. 1-2, pp. 64-66, 1950.

[4] T. M. Rassias, "On the stability of the linear mapping in Banach spaces," Proceedings of the American Mathematical Society, vol. 72, no. 2, p. 297, 1978.

[5] P. Gavruta, "A generalization of the Hyers-Ulam-Rassias stability of approximately additive mappings," Journal of Mathematical Analysis and Applications, vol. 184, no. 3, pp. 431-436, 1994.

[6] J. Erdős, "A remark on the paper on some functional equationsby S. Kurepa," Matematičko-Fizički i Astronomski Glasnik, vol. 14, pp. 3-5, 1959.

[7] B. Jessen, J. Karpf, and A. Thorup, "Some functional equations in groups and rings," Mathematica Scandinavica, vol. 22, pp. $257-265,1968$.

[8] S. A. Mohiuddine, J. M. Rassias, and A. Alotaibi, "Solution of the Ulam stability problem for Euler-Lagrange 
( $\alpha, \beta ; k)$-quadratic mappings," Journal of Computational Analysis and Applications, vol. 20, no. 2, pp. 363-373, 2016.

[9] S. A. Mohiuddine, J. Rassias, and A. Alotaibi, "Solution of the Ulam stability problem for Euler-Lagrange-Jensen k-cubic mappings," Filomat, vol. 30, no. 2, pp. 305-312, 2016.

[10] S. A. Mohiuddine, J. M. Rassias, and A. Alotaibi, "Solution of the Ulam stability problem for Euler-Lagrange-Jensenkquintic mappings," Mathematical Methods in the Applied Sciences, vol. 40, no. 8, pp. 3017-3025, 2017.

[11] D. G. Bourgin, "Approximately isometric and multiplicative transformations on continuous function rings," Duke Mathematical Journal, vol. 16, no. 2, pp. 385-397, 1949.

[12] G. Maksa and Z. Páles, "Hyperstability of a class of linear functional equations," Acta Mathematica, vol. 17, no. 2, pp. 107-112, 2001.

[13] M. Almahalebi, A. Charifi, and S. Kabbaj, "Hyperstability of a Cauchy functional equation," Journal Numerical Functional Analysis and Optimization, vol. 6, no. 2, pp. 127-137, 2015.

[14] M. Almahalebi, "On the hyperstability of $\sigma$-Drygas functional equation on semigroups," Aequationes Mathematicae, vol. 90, no. 4, pp. 849-857, 2016.

[15] M. Almahalebi and C. Park, "On the hyperstability of a functional equation in commutative groups," Journal of Computational Analysis and Applications, vol. 20, no. 1, pp. 826-833, 2016.

[16] M. Almahalebi and A. Chahbi, "Hyperstability of the Jensen functional equation in ultrametric spaces," Aequationes Mathematicae, vol. 91, no. 4, pp. 647-661, 2017.

[17] A. Alotaibi, J. M. Rassias, and S. A. Mohiuddine, "Solution of the Ulam stability problem for quartic $(a, b)$-functional equations," Journal of Computational Analysis and Applications, vol. 20, no. 5, pp. 957-968, 2016.

[18] Y. Aribou, M. Almahalebi, and S. Kabbaj, "Hyperstability of cubic functional equation in ultrametric spaces," Proyecciones (Antofagasta), vol. 36, no. 3, pp. 461-484, 2017.

[19] Y. Aribou, H. Dimou, and S. Kabbaj, "The generalized hyperstability of cubic functional equation," Palestine Journal of Mathematics, vol. 9, no. 1, pp. 485-492, 2020.

[20] A. Bahyrycz and M. Piszczek, "Hyperstability of the Jensen functional equation," Acta Mathematica Hungarica, vol. 142, no. 2, pp. 353-365, 2014.

[21] J. Brzdęk, "Hyperstability of the Cauchy equation on restricted domains," Acta Mathematica Hungarica, vol. 141, no. 1-2, pp. 58-67, 2013.

[22] J. Brzdęk, "Remarks on hyperstability of the Cauchy functional equation," Aequationes Mathematicae, vol. 86, no. 3, pp. 255-267, 2013.

[23] J. Brzdęk, "A hyperstability result for the Cauchy equation," Bulletin of the Australian Mathematical Society, vol. 89, no. 1, pp. 33-40, 2014.

[24] J. Brzdęk, J. Chudziak, and Zs. Páles, “A fixed point approach to stability of functional equations," Nonlinear Analysis, vol. 74, no. 17, pp. 6728-6732, 2011.

[25] J. Brzdęk and K. Ciepliński, "A fixed point approach to the stability of functional equations in non-Archimedean metric spaces," Nonlinear Analysis, vol. 74, no. 18, pp. 6861-6867, 2011.

[26] J. Brzdęk and K. Ciepliński, "Hyperstability and superstability," Abstract and Applied Analysis, vol. 2013, Article ID 401756, 13 pages, 2013.

[27] E. Gselmann, "Hyperstability of a functional equation," Acta Mathematica Hungarica, vol. 124, no. 1-2, pp. 179-188, 2009.
[28] M. Piszczek, "Remark on hyperstability of the general linear equation," Aequationes Mathematicae, vol. 88, no. 1-2, pp. 163-168, 2014.

[29] M. Sirouni and S. Kabbaj, "A fixed point approach to the hyperstability of Drygas functional equation in metric spaces," Journal of Mathematical and Computational Science, vol. 4, no. 4, pp. 705-715, 2014.

[30] A. Khrennikov, Non-Archimedean Analysis, Quantum Paradoxes, Dynamical Systems and Biological Models, Kluwer Academic Publishers, Dordrecht, Netherlands, 1997.

[31] A. Bahyrycz, J. Brzdek, and M. Piszczek, "On approximately p-wright affine functions in ultrametric spaces," Journal of Function Spaces and Applications, vol. 2013, pp. 1-7, 2013.

[32] A. Bahyrycz and J. Olko, "Stability of the equation of $(p, q)$ Wright functions," Acta Mathematica Hungarica, vol. 146, no. 1, pp. 71-85, 2015.

[33] A. Bahyrycz and J. Olko, "On stability of the general linear equation," Aequationes Mathematicae, vol. 89, no. 6, pp. 1461-1474, 2015.

[34] D. Zhang, "On Hyers-Ulam stability of generalized linear functional equation and its induced Hyers-Ulam programming problem," Aequationes Mathematicae, vol. 90, no. 3, pp. 559-568, 2016.

[35] D. Zhang, "On hyperstability of generalised linear functional equations in several variables," Bulletin of the Australian Mathematical Society, vol. 92, no. 2, pp. 259-267, 2015.

[36] J. Brzdęk, "Stability of additivity and fixed point methods," Fixed Point Theory and Applications, vol. 2013, p. 285, 2013. 